\title{
Dust and spacecraft charging in Saturn's E ring
}

\author{
Hsiang-Wen Hsu, Mihály Horányi, and Sascha Kempf \\ Laboratory of Atmospheric and Space Physics, University of Colorado at Boulder, CO 80309
}

(Received November 7, 2011; Revised May 27, 2012; Accepted May 29, 2012; Online published March 12, 2013)

\begin{abstract}
The electrostatic potential of a spacecraft reflects the local physical conditions and also affects the in situ thermal plasma measurements. In this work we focus on the spacecraft charging in the high dust density region near Enceladus in Saturn's E ring. In a dust-rich environment, the spacecraft charging is modified by the complex (dusty) plasma conditions as well as by the additional dust-associated charging currents (dust ram current and the dust impact plasma current). Adopting the Cassini plasma electron and dust profiles, we explore the longitudinal and latitudinal spacecraft potential variations in the E ring. Our results show that, under dust-rich condition, the spacecraft potential is neutralized due to the collection of the dust impact plasma ions. We suggest a systematic verification of our model calculation with the Cassini in situ measurements and also a search for signatures of dust-spacecraft impact plasma in the Cassini thermal plasma measurements.
\end{abstract}

Key words: Spacecraft charging, dust, Saturn, Enceladus, E ring, Cassini mission.

\section{Introduction}

The charging of a surface in space is determined by the collection of ambient plasma as well as the emission of photoelectrons and/or secondary electrons (Whipple, 1981). Hence, the charging status of an object varies with various physical conditions as well as with its material properties.

The diffuse E ring in Saturn's magnetosphere is an ideal laboratory for studying the charging processes of dust and spacecraft. Fed by ice particles from the volcanically active moon Enceladus (Hansen et al., 2006; Porco et al., 2006; Spahn et al., 2006; Waite et al., 2006), the E ring spans from about $3 \mathrm{R}_{\mathrm{S}}\left(\mathrm{R}_{\mathrm{S}}=60268 \mathrm{~km}\right.$ is the Saturn radius) to Titan's orbit at $20 \mathrm{R}_{\mathrm{S}}$ (Srama et al., 2006) in Saturn's magnetosphere. The plasma conditions experienced by the ring particles vary from the cold plasma at the dense Enceladus torus to the hot, tenuous outer magnetospheric plasma. In a dense Maxwellian plasma where the photoemission can be ignored, an object will be charged negatively due to the dominant electron collection current. Based on the dust electrostatic charge measurements carried out by the Cassini Cosmic Dust Analyser (CDA, Srama et al., 2004) in the E ring, Kempf et al. (2006) showed that the dust potential is about $-2 \mathrm{~V}$ near Enceladus $\left(4 \mathrm{R}_{\mathrm{S}}\right)$ and becomes positive approximately outside the orbit of the moon Rhea $\left(8.7 R_{S}\right)$. The grain charge measurements are roughly matched by the charging calculation using Cassini plasma measurements. The results show that in the dense and cold Enceladus torus the electron collection is the dominant charging current, which leads to a negative dust potential. In contrast, the hotter electron population at larger Saturn distance produces secondary electrons that charges

Copyright (C) The Society of Geomagnetism and Earth, Planetary and Space Sciences (SGEPSS); The Seismological Society of Japan; The Volcanological Society of Japan; The Geodetic Society of Japan; The Japanese Society for Planetary Sciences; TERRAPUB

doi:10.5047/eps.2012.05.018 dust positively in the middle to outer magnetosphere. The radial grain potential profile obtained by CDA clearly reflects the variation of the plasma condition in Saturn's magnetosphere.

The charging profile of the Cassini spacecraft is found to be similar to the E ring dust. During Cassini's insertion into its Saturnian orbit in July 2004, data obtained by the Cassini Langmuir probe/Radio and Plasma Wave Science instrument (LP/RPWS, Gurnett et al., 2004) show that the spacecraft is charged a few volts negatively within $10 \mathrm{R}_{\mathrm{S}}$ (Wahlund et al., 2005). Outside $\sim 8 \mathrm{R}_{\mathrm{S}}$ where the spacecraft is charged positively, some of the photoelectrons induced from the solar ultraviolet radiation are drawn back to the spacecraft and are identified in the Cassini Plasma Spectrometer (CAPS, Young et al., 2004) electron data (Lewis et al., 2008). The derived spacecraft potential is consistent with the LP results (Sittler et al., 2005, 2006).

Recently, in addition to the radial variation across the magnetosphere, more attention are focused on the inner region near Enceladus. Several magnetospheric phenomena are found to be organized with the rotation of Saturn, defined by the periodic Saturn Kilometric Radiation (SKR, Warwick et al., 1981). For example, Gurnett et al. (2007) demonstrated that the electron density between 3 to $5 \mathrm{R}_{\mathrm{S}}$ varies with the SKR longitude system by a factor of two. Also, in the dust-rich environment near Enceladus, a series of studies based on the Cassini LP measurements suggest that the electron density is far less than the ion density. This electron depletion is attributed to the electron attachment to the abundant sub-micron-sized dust particles, and has been suggested as evidence for collective dust-plasma interactions (Farrell et al., 2009, 2010; Wahlund et al., 2009; Yaroshenko et al., 2009; Morooka et al., 2011; Shafiq et al., 2011).

Depending on their abundance, the presence of dust grains in a plasma could have significant effects on the 
charging processes. With increasing dust density in a dustplasma cloud, more and more plasma electrons will be collected by the dust. This leads to the depletion of plasma electrons and a decreasing potential of the dust-plasma cloud $\left(\phi_{c}\right)$ (Havnes et al., 1990). As most charging currents depend on the potential difference between the object and $\phi_{c}\left(\Phi_{i}=\phi_{i}-\phi_{c}\right.$, where $i=d$ or $S C$ for dust and spacecraft, respectively), the negative shift of this charging baseline modifies the potential of embedded objects (Goertz and Ip, 1984; Havnes et al., 1990). Moreover, considering a spacecraft traversing through a dust-rich region, the intense dust flux received by the spacecraft constitutes additional charging currents to the spacecraft. In our earlier work (Hsu et al., 2012), we considered two types of dust currents, the dust ram current and dust impact plasma current (see Section 2), to model the spacecraft potential under dust-rich conditions. This model better reproduces the Cassini spacecraft potential measurements in the E ring and explains the difference between the Cassini LP potential measurements and the Particle-in-cell (PIC) simulations by Olson et al. (2010) implying that the spacecraft potential near Enceladus reflects not only the plasma but also the dust conditions.

Following our earlier work, we extend the spacecraft potential calculation to understand the influence of $E$ ring dust on the longitudinal and latitudinal variations of the spacecraft potential near Enceladus at $4 \mathrm{R}_{\mathrm{S}}$. In Section 2 we describe the currents considered in the spacecraft charging calculation. The plasma and dust properties in the E ring are described in Section 3. Modeling results and the discussion are presented in Section 4. A brief summary is given in Section 5.

\section{Charging Current Calculation}

A widely used method to model the charging of an object in a plasma is the Orbital Motion Limited (OML) theory (Mott-Smith and Langmuir, 1926; Whipple, 1981; Horányi, 1996). The OML theory treats the collection of plasma particles as a two-body problem, which can be solved by considering the conservation of energy and angular momentum.

\subsection{Electron and ion collection currents}

Following the OML theory, the collection currents of electrons and ions with Maxwellian energy distribution can be written as

$$
J_{e, i}=J_{e, i}^{0} \times\left\{\begin{array}{ll}
1-\chi_{e, i}, & \text { if } \chi_{e, i}<0 \\
e^{-\chi_{e, i},} & \text { if } \chi_{e, i} \geq 0
\end{array},\right.
$$

where $J_{e, i}^{0}=\mp 4 A_{\mathrm{sc}} e n_{e, i}\left(k T_{e, i} / 2 \pi m_{e, i}\right)^{1 / 2}, \quad \chi_{e, i}=$ $\mp e \Phi_{\mathrm{sc}} / k T_{e, i}, k$ is Boltzmann's constant, and $e$ is the elementary charge. $\Phi_{\mathrm{sc}}=\phi_{\mathrm{sc}}-\phi_{c}$ is the potential difference between the spacecraft $\left(\phi_{\mathrm{sc}}\right)$ and the dust-plasma cloud $\left(\phi_{c}\right)$. $A_{\mathrm{sc}}$ is the effective surface area of the spacecraft. The $n_{e, i}$, $k T_{e, i}$, and $m_{e, i}$ are the density, temperature, and mass of electrons and ions, respectively. We consider the plasma ions to be singly ionized oxygen $\left(\mathrm{O}^{+}\right)$.

Due to the low ion temperature in the vicinity of Enceladus, the plasma ion flux to the spacecraft is not isotropic as the average ion thermal speed is less than the corotation flow speed (see Subsubsection 3.1.1). This effect has been considered in this work for the spacecraft charging calculation (Whipple, 1981; Horányi, 1996; and references therein).

\subsection{Photoelectron emission}

Photoelectron emission from the spacecraft is

$$
J_{v}=J_{v}^{0} \times \begin{cases}1, & \text { if } \chi_{v} \leq 0 \\ e^{-\chi_{v}}, & \text { if } \chi_{v}>0\end{cases}
$$

where $J_{v}^{0}=A_{\mathrm{sc}} e \kappa_{v} f_{d}, \chi_{v}=-e \Phi_{\mathrm{sc}} / k T_{v}$, and $\kappa_{v}$ is the photoelectric coefficient. We adopt $\kappa_{v}=0.1$ for icy dust grains and 0.5 for the spacecraft. $f_{d}=2.5 \times 10^{14} d_{\mathrm{AU}}^{-2}$ $\mathrm{m}^{-2} \mathrm{sec}^{-1}$ is the solar UV photon flux at the distance $d_{\mathrm{AU}}$ (AU) from the Sun. The energy of photo-electrons follows the Maxwellian distribution with a temperature of $k T_{v}=$ $3 \mathrm{eV}$ (Grard and Tunaley, 1971; Horányi, 1996).

\subsection{Dust ram current}

The dust ram current is the charge flux carried from dust to the spacecraft:

$$
J_{d, \mathrm{ram}}=\int_{s_{\min }}^{s_{\max }} Q_{d}\left(s_{d}\right) \cdot f_{d}\left(s_{d}, v_{\mathrm{rel}}\right) d s_{d},
$$

(Hsu et al., 2012), where $s_{d}$ is the dust radius, $Q_{d}\left(s_{d}\right)=$ $4 \pi \epsilon_{0} s_{d} \Phi_{d}$ is the dust charge, and $\Phi_{d}=\phi_{d}-\phi_{c}$ is the potential difference between dust $\left(\phi_{d}\right)$ and the background dust-plasma cloud. $f_{d}\left(s_{d}, v_{\text {rel }}\right)=A_{\text {sc }} \cdot v_{\text {rel }}\left(s_{d}\right) \cdot n_{d}\left(s_{d}\right)$ is the dust flux to the spacecraft. $v_{\text {rel }}$ is the relative speed between dust and the spacecraft and varies with the dust size (see Subsubsection 3.2.2). We adopt a power-law dust size distribution $n_{d} d s_{d}=n_{d 0} \cdot\left(s_{d} / 1 \mu \mathrm{m}\right)^{-\alpha}$, where $n_{d 0}$ is the density of $1 \mu \mathrm{m}$ dust and $\alpha$ is the power-law slope. Here we choose $s_{\max }=10 \mu \mathrm{m}, s_{\min }=0.05 \mu \mathrm{m}$, and $\alpha=5$. It is worthy to mention that the dust ram current does not depend on the spacecraft potential as the kinetic energy of dust is much larger than the electrostatic potential energy of the spacecraft.

\subsection{Dust impact plasma current}

When a dust grain hits the spacecraft at high speed, part of the dust kinetic energy is transformed to ionize the impactor (dust) and the target (spacecraft), i.e., the so-called impact ionization process (Hornung and Drapatz, 1979). From the initial dense state the impact plasma cloud expands until being confined by the magnetic field or dispersing into the ambient plasma in a timescale of a microsecond. Due to the spacecraft charge, part of the impact plasma will be collected by the spacecraft depending on their kinetic energy as well as on its electric polarity. This constitutes a charging current- the dust impact plasma current-which actually consists of two components: the electron collection and ion collection currents.

The empirical impact plasma production yield $(Y$, in Coulombs) for an impactor with mass of $m_{d}$ and impact speed of $v_{\text {rel }}$ is $Y=5 \cdot 10^{-4} \cdot m_{d} \cdot v_{\text {rel }}^{3.5}$ (Dietzel et al., 1973; Göller and Grün, 1989). The impact plasma flux to the spacecraft is thus

$$
S_{\mathrm{imp}}=\int_{s_{\min }}^{s_{\max }} f_{d}^{m}\left(s_{d}, \varrho_{d}, v_{\mathrm{rel}}\right) \cdot Y\left(s_{d}, \varrho_{d}, v_{\mathrm{rel}}\right) d s_{d},
$$

where $f_{d}^{m}\left(s_{d}, \varrho_{d}, v_{\text {rel }}\right)=A_{\mathrm{sc}} \cdot m_{d}\left(\varrho_{d}, s_{d}\right) \cdot v_{\text {rel }}\left(s_{d}\right) \cdot n_{d}\left(s_{d}\right)$ is the dust mass flux and $\varrho_{d}=10^{3} \mathrm{~kg} \mathrm{~m}^{-3}$ is the dust bulk density. 
The collection of the impact plasma depends on its energy distribution and the spacecraft potential. As indicated by the ion line shape from the CDA dust mass spectra, an expanding impact plasma cloud is relatively cold and most probably inherit the LTE properties from the initial hot, dense state. Hence, it can be described as a mixture of the same amount of ions and electrons, whose energy distributions are Maxwellian with a characteristic temperature, $k T_{\text {imp }}$, of $\sim 1 \mathrm{eV}$ (Kempf et al., 2012). Following Hsu et al. (2012), the dust impact-plasma current becomes

$$
J_{d, \text { imp }}^{e, i}=S_{\text {imp }} \times\left\{\begin{array}{ll}
1, & \text { if } \chi_{e, i}^{\text {imp }} \leq 0 \\
\exp \left(-\chi_{e, i}^{\mathrm{imp}}\right), & \text { if } \chi_{e, i}^{\mathrm{imp}}>0
\end{array},\right.
$$

where $\chi_{e, i}^{\mathrm{imp}}=\mp e \Phi_{\mathrm{sc}} / k T_{\mathrm{imp}}$.

The number of ions and electrons that the spacecraft collects from impact plasma depends on the charge polarity. A negatively charged spacecraft will collect more impact plasma ions and vice versa. The impact plasma hence acts as a "potential damper" and neutralizes the spacecraft surface.

Near Enceladus, the Cassini-dust relative speed is typically faster than a few $\mathrm{km} \mathrm{s}^{-1}$. Compared to the charges carried on dust grains $\left(\Phi_{d} \sim 2 \mathrm{~V}\right)$, the impact plasma may strongly affect the spacecraft potential. For conditions studied in this work, the dust impact plasma currents are more important than the dust ram current.

\subsection{Charge equilibrium}

The time-varying spacecraft charge $\left(Q_{\mathrm{sc}}\right)$ can be solved by integrating the charging currents Eqs. (1)-(5):

$$
\frac{d Q_{\mathrm{sc}}}{d t}=\sum J\left(\Phi_{\mathrm{sc}}\right) \text {. }
$$

All currents described so far but the dust ram current depend on the spacecraft-dust-plasma cloud potential difference $\left(\Phi_{\mathrm{sc}}\right)$. Given enough time, the spacecraft will reach the charge equilibrium, i.e., $\Phi_{\mathrm{sc}}=\Phi_{\mathrm{sc}}^{\mathrm{eq}}$ where $\sum J\left(\Phi_{\mathrm{sc}}^{\mathrm{eq}}\right)=0$. The time to reach charge equilibrium depends on the magnitude of currents. Without dust currents, the charging time is about $Q_{\mathrm{sc}}^{\mathrm{eq}} / J_{e}$ (where $Q_{\mathrm{sc}}^{\mathrm{eq}}$ is the spacecraft equilibrium charge), as the electron collection current always dominates in the dense and cold plasma torus of Enceladus.

Assuming a simplified spherical spacecraft with a radius of 6 meters, the spacecraft charge, $Q_{\mathrm{sc}}^{\mathrm{eq}}$, is of the order of $10^{-9} \mathrm{C}$. Considering an electron density of $100 \mathrm{~cm}^{-3}$ and an electron temperature of $1 \mathrm{eV}$, the electron flux to the spacecraft is about $10^{-3} \mathrm{Cs}^{-1}$ (or $10^{16}$ electrons per second) and leads to a charging timescale of $\sim 10^{-6}$ second.

In contrast to the macroscopically continuous plasma collection, dust impacts, and hence dust currents, are sporadic by nature. However, near Enceladus, the dust impact rate is so high that it is reasonable to approximate the dust currents as a continuous process. For example, with a dust density of $0.1 \mathrm{~m}^{-3}$ and $v_{\text {rel }}=10 \mathrm{~km} \mathrm{~s}^{-1}$, the dust-spacecraft impact rate is about $10^{5}$ per second. The expansion time of the impact plasma is also short enough to be ignorable. We thus expect the spacecraft charging time to be much shorter than one second.

In short, near Enceladus the spacecraft adjusts almost instantly to the equilibrium potential, with or without dust currents. In the discussion below, we will use the calculated equilibrium potential to represent the spacecraft potential.

\subsection{Dusty plasma conditions}

The dust-plasma cloud potential shifts toward negative values in dust-rich conditions. In this work we follow the scheme by Havnes et al. (1990) and describe the potentials of the dust-plasma cloud and the embedded dust as functions of the $P$ parameter:

$$
P=6.95 \cdot 10^{6} \cdot n_{e, 0}^{-1} \cdot k T_{e} \cdot \int_{s_{\min }}^{s_{\max }} s_{d} \cdot n_{d}\left(s_{d}\right) d s_{d},
$$

where $n_{e, 0}$ is the background electron density and $N_{d}=$ $\int n_{d}\left(s_{d}\right) d s_{d} \mathrm{~m}^{-3}$ is the total dust density. The $P$ parameter is the ratio of the charge density in the dust component, calculated for isolated dust particles, relative to the background electron density. The normalized dust potential $\left(e \Phi_{d} / k T_{e}\right)$ and the cloud $\left(e \phi_{c} / k T_{e}\right)$ is shown in Fig. 1 as functions of $P$. When $P \ll 1$, the dust can be seen as a single grain in the plasma. With increasing $P$, the inter-grain distance decreases and the Debye sphere of individual dust particles overlaps, which results in mutual shielding effect and the reduction of dust charges. In addition, the dust-plasma cloud potential also affects the densities of electrons and ions: $n_{e}=n_{e, 0} \cdot \exp \left(-e \phi_{c} / k T_{e}\right)$ and $n_{i}=n_{i, 0} \cdot \exp \left(e \phi_{c} / k T_{i}\right)$, where $n_{e, 0}=n_{i, 0}$ are the background electron and ion density.

\section{Plasma and Dust Distributions}

\subsection{Magnetospheric plasma}

3.1.1 Water-group ions The main ion population in Saturn's magnetosphere is water-group ions, the dissociated and ionized products of water vapor emitted from Enceladus. Our model adopts $\mathrm{O}^{+}$for the ion population. The ion temperature near Enceladus is found to be about $25 \mathrm{eV}$ (Sittler et al., 2005), corresponding to a thermal speed of $16 \mathrm{~km} \mathrm{~s}^{-1}$ and is small compared to the co-rotating flow (30-40 $\mathrm{km} \mathrm{s}^{-1}$ ) energy. Hence, the thermal component of the ion collection is negligible. In our simulation, we simply assume that the energy distribution of both ions and electrons are Maxwellian and that their density and temperature are the same $\left(n_{i, 0}=n_{e, 0}\right.$ and $\left.T_{i}=T_{e}\right)$.

3.1.2 Thermal electrons Gurnett et al. (2007) observed a sinusoidal electron density $\left(N_{e, \lambda_{\mathrm{SC}}}\right)$ modulation along the SKR longitude system (SLS) between 3 to $5 R_{S}$. We adopt the following description for the electron density near Enceladus $\left(4 R_{S}\right)$ in the ring plane:

$$
N_{e, \lambda_{\mathrm{SC}}}=65+25 \times \sin \left(\lambda_{\mathrm{SC}}-240^{\circ}\right) \mathrm{cm}^{-3} .
$$

$\lambda_{\mathrm{SC}}$ is the SKR longitude of the spacecraft. Moreover, the electron density also decreases with distance from the equatorial plane. Combining this with Eq. (8), the electron density is

$$
N_{e, z}=N_{e, \lambda \mathrm{SC}} \times e^{-z^{2} / H_{n}^{2}} \mathrm{~cm}^{-3}
$$

where $H_{n}=0.5 \mathrm{R}_{\mathrm{S}}$ is the electron density scale height (Persoon et al., 2006).

The Cassini LP measurements indicate that the electron temperature at $4 \mathrm{R}_{\mathrm{S}}$ is about $2.3 \mathrm{eV}$ and increases toward 


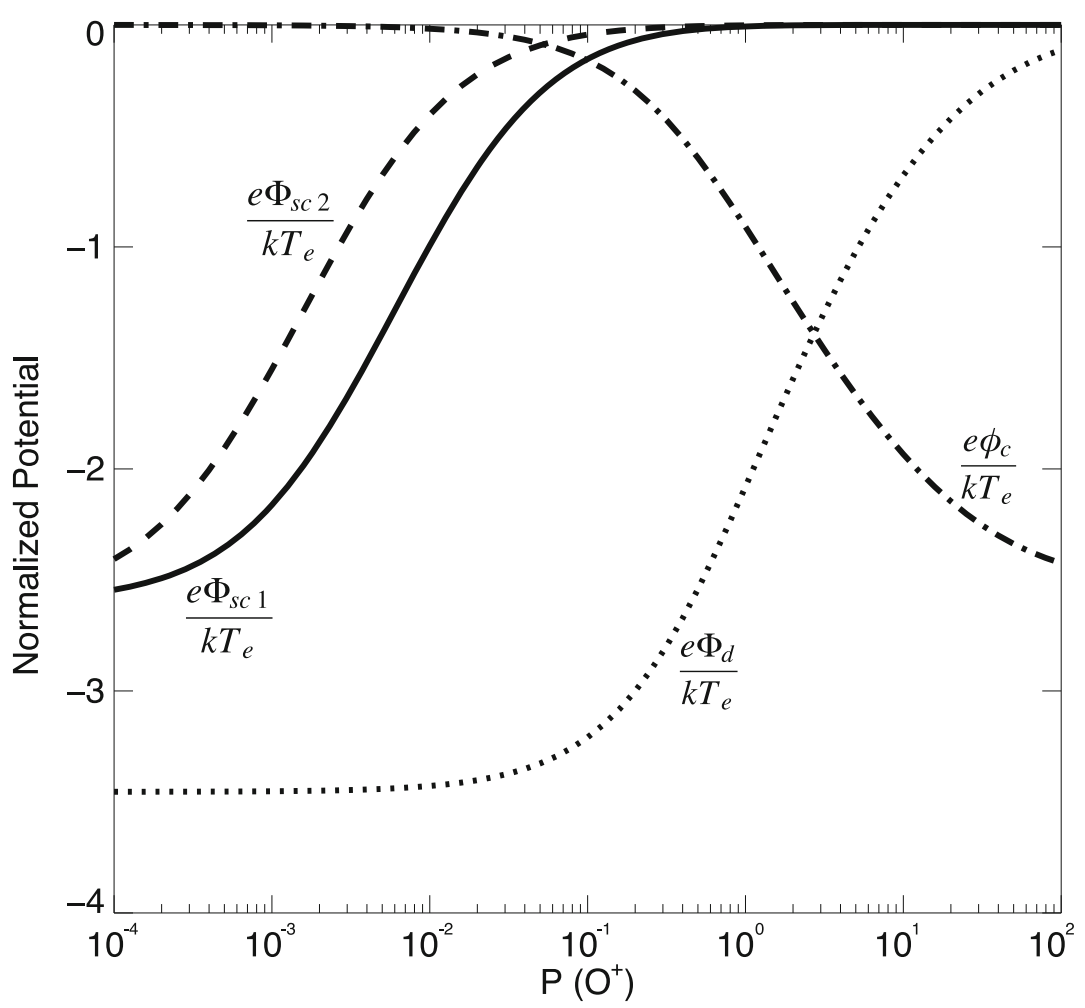

Fig. 1. The normalized potential profiles of the dust-plasma cloud ( $e \phi_{c} / k T_{e}$, dot-dash line), the embedded dust ( $e \Phi_{d} / k T_{e}$, dotted line), and the spacecraft as functions of $P$. Two spacecraft potential profiles represent cases with different speeds: $8 \mathrm{~km} \mathrm{~s}^{-1}\left(e \Phi_{\mathrm{sc} 1} / k T_{e}\right.$, solid line $)$ and $16 \mathrm{~km} \mathrm{~s}^{-1}\left(e \Phi_{\mathrm{sc} 2} / k T_{e}\right.$, dash line). With increasing $P$ the SC potential becomes less negative because of the reduction of free electron density as well as the ion collection from the impact-generated plasma.

higher latitude

$$
T_{e, Z}=2.3 \cdot e^{Z^{2} / H_{T}^{2}} \mathrm{eV}
$$

where $Z$ is the distance to the ring plane in $\mathrm{R}_{\mathrm{S}}$ and $H_{T} \sim$ $0.8 \mathrm{R}_{\mathrm{S}}$ is the electron temperature scale height (Gustafsson and Wahlund, 2010).

\subsection{E ring}

3.2.1 Dust number density profile We adopt the dust density model based on the CDA measurements between 2005 and 2006 (Kempf et al., 2008). This model is symmetric with respect to Saturn and the ring plane. The density for grains larger than $0.9 \mu \mathrm{m}$ at $4 \mathrm{R}_{\mathrm{S}}$ is $N_{d, 0}=$ $0.12 \mathrm{~m}^{-3}$. Away from the ring plane, the dust density decreases exponentially

$$
N_{d, z}=N_{d, 0} \cdot e^{-z^{2} / 2 \sigma^{2}}
$$

where $\sigma=1839 \mathrm{~km}$ is the full-width-half-maximum of the E ring at $4 \mathrm{R}_{\mathrm{S}}$ and $z=Z \cdot \mathrm{R}_{\mathrm{S}}$ in $\mathrm{km}$.

3.2.2 Dust speed distribution Due to the Lorentz force, the speed of charged dust depends on its charge-tomass ratio. Assuming a circular orbit in the magnetic equator, the angular velocity $(\psi)$ of a dust particle with a chargeto-mass ratio, $Q_{d} / m_{d}$, at a distance to the planet, $R$, can be found by solving (equation (22) in Horányi, 1996)

$$
\psi^{2}+\omega_{g} \psi-\omega_{g} \Omega-\omega_{k}^{2}=0
$$

where $\omega_{g}=Q_{d} / m_{d} B_{z}$ is the local dust gyrofrequency and $\omega_{k}=\sqrt{G M_{p} / R^{3}}$ is the Kepler angular velocity. The rotation rate and the mass of the planet are $\Omega$ and $M_{p}$, respectively. $B_{z}$ is the local magnetic field strength and $G$ is the gravitational constant. The dust speed at $4 \mathrm{R}_{\mathrm{S}}$ as a function of dust radius is shown in Fig. 2.

\section{Results and Discussions}

Taking all currents into account, we first compare the potential of the spacecraft, the dust, and the dust-plasma cloud for different values of $P$ in Fig. 1 for two flyby speeds. When $P \ll 1$, both cases show the potential about $0.8 \mathrm{~V}$ less negative than the dust because of the higher photoemission efficiency. With increasing $P$, the dust currents, especially the dust impact plasma current, becomes more and more important. Since the spacecraft is charged negatively, it collects more ions than electrons from the impact plasma, which results in a positive shift of the spacecraft potential. The strong velocity dependence of the impact plasma production Eq. (4) leads to a faster rising potential for the spacecraft with higher $v_{\text {rel }}$.

\subsection{Longitudinal and latitudinal variations}

Figures 3 and 4 show the longitudinal and latitudinal spacecraft potential variation at $4 \mathrm{R}_{\mathrm{S}}$, respectively. The longitudinal potential variation shown in Fig. 3 results from the electron density modulation along the SLS longitude (Eq. (8) and Fig. 3(c)). In general, the spacecraft is charged most negatively at $\lambda_{\mathrm{SC}}=330^{\circ}$, where it encounters the highest electron density. When no dust is present (i.e., only $J_{e}, J_{i}$, and $J_{v}$ are considered), the spacecraft is charged to $-7 \mathrm{~V}$ with about $0.5 \mathrm{~V}$ fluctuation depending on the spacecraft longitude. With dust, the potential profiles show a sim- 


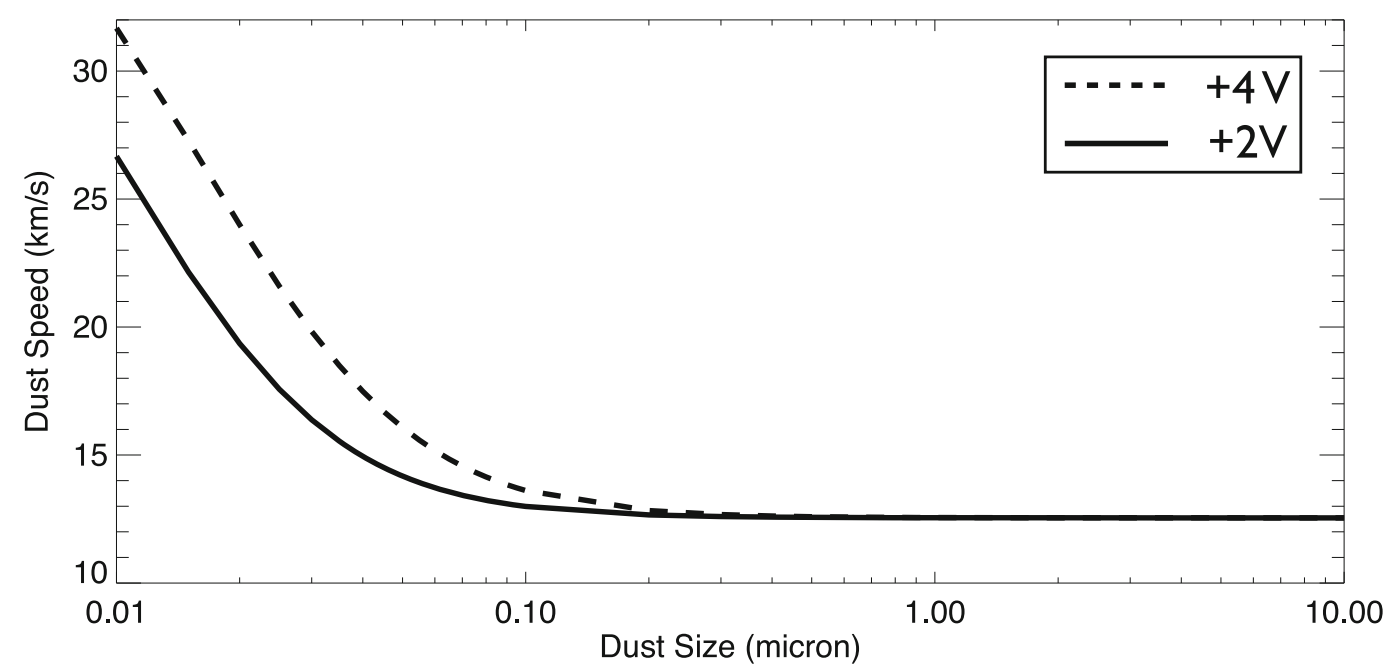

Fig. 2. The dust speed as a function of the size at $4 \mathrm{R}_{\mathrm{S}}$. Two profiles represent dust with potential of $+2 \mathrm{~V}$ (solid line) and $+4 \mathrm{~V}$ (dash line). Dust grains larger than 0.5 micron follow the Keplerian motion while smaller particles have faster speed due to the influence of the Lorentz force.

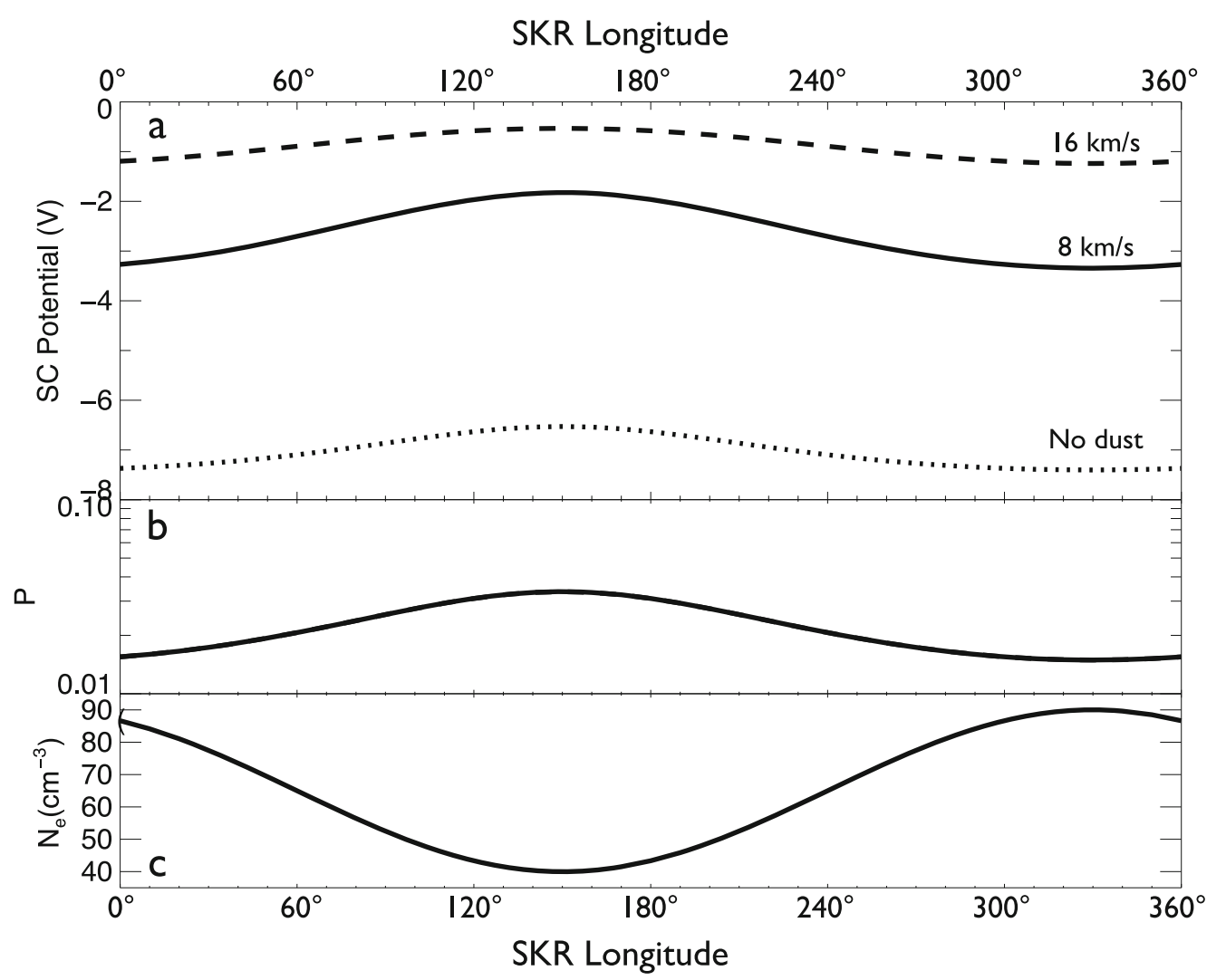

Fig. 3. The variations of (a) the spacecraft potential, (b) the $P$ parameter, and (c) the electron density along the SKR longitude. Three spacecraft potential profiles represent the cases of zero dust current (dotted line), $8 \mathrm{~km} \mathrm{~s}^{-1}$ spacecraft speed (solid line), and $16 \mathrm{~km} \mathrm{~s}{ }^{-1}$ speed (dash line). All profiles fluctuate along the SKR longitude due to the electron density modulation. Compared to the case with no dust current, the spacecraft potential is shifted positively. The faster the spacecraft speed, the less negative the potential becomes.

ilar trend as the dust-free case, except that the potential is shifted toward less negative values. As is the case in Fig. 1, the faster spacecraft receives a larger flux of impact plasma ions, resulting in a stronger reduction of the spacecraft potential. The $P$ parameter here is about 0.02 (Fig. 3(b)), which means that the dust-plasma cloud and dust potentials are only slightly modified (Fig. 1).
The condition for a steep ring plane crossing (RPX) at $4 \mathrm{R}_{\mathrm{S}}$ is shown in Fig. 4. We set the electron density at the ring plane to be $75 \mathrm{~cm}^{-3}$ and assume its decrease is given by Eq. (10). The latitudinal profiles of the dust number density as well as the plasma electron temperature are shown in Figs. 4(b) and 4(c). The parameters for calculating the profiles shown in Fig. 4(a) are the same as those in Fig. 3(a). 


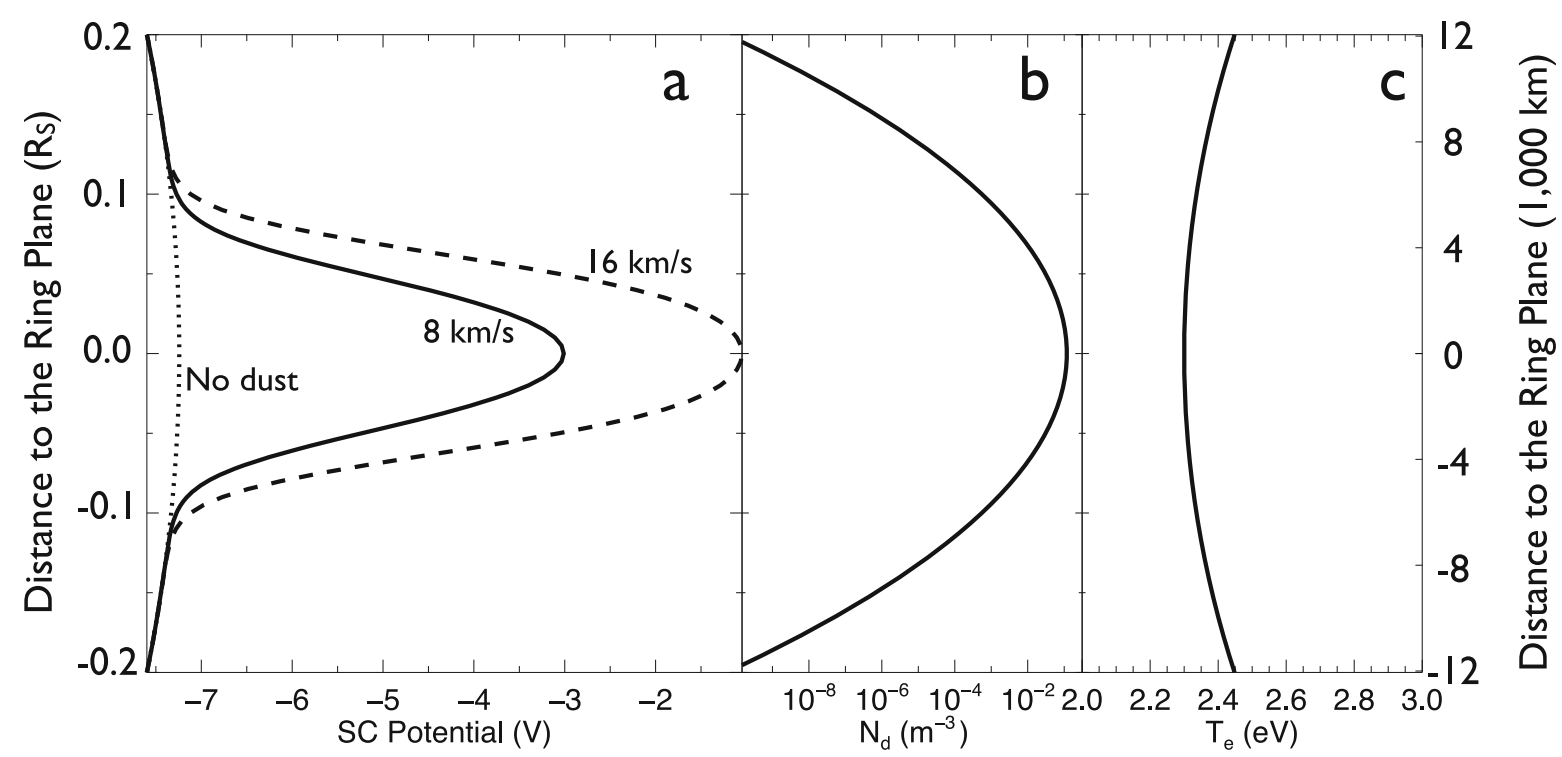

Fig. 4. Latitudinal variations of (a) the spacecraft potential, (b) the density of dust with $s_{d}>0.9 \mu \mathrm{m}$, and (c) the electron temperature. Three spacecraft profiles shown in (a) represent the same conditions as those in Fig. 3(a). Because of the concentration of dust particles near the ring plane, the spacecraft is charged less negatively at lower latitude.

If the dust effects are ignored (dotted line), the spacecraft potential becomes a little less negative at the ring plane due to the lower electron temperature. As shown in Fig. 4(b), the dust density is much lower at higher latitudes, the influence of dust currents is thus negligible when $|z|>0.1 \mathrm{R}_{\mathrm{S}}$. Within $0.1 \mathrm{R}_{\mathrm{S}}$ from the ring plane, the "dusty" cases (solid and dash lines) deviate from the dust-free case (dotted line) and reach more positive potentials at lower latitude. As shown before, the higher the spacecraft speed, the larger the spacecraft potential shift toward the dust-plasma cloud potential.

\subsection{Discussion}

It becomes apparent in Figs. 3 and 4 that the spacecraft potential in the dusty conditions cannot be modeled precisely without knowing the spacecraft speed and the plasma and dust properties. Each component plays an important role in determining the potential of the spacecraft. The influence of the dust currents depends on the dust density as well as the relative speed between dust and spacecraft. The example shown in Fig. 3 indicates that the influence of dust currents on the spacecraft charging $\left(\mathrm{a}+5 \mathrm{~V} \Phi_{\mathrm{sc}}\right.$ shift $)$ is more significant than the effect caused by the electron density modulation in the ring plane (a $0.5 \mathrm{~V} \Phi_{\mathrm{sc}}$ fluctuation). However, if the true dust density is not longitudinally uniform as assumed here, the spacecraft potential may not exhibit a clear correlation with the SKR longitude as the plasma electron density.

Our results can be verified by adopting both in-situ plasma and dust measurements to the model and comparing results with the spacecraft potential measurements. However, to make a meaningful comparison the temporal and spacial variations of the parameters, as well as the spacecraft attitude, need to be considered in greater detail for each RPX. There are several factors that may change along the spacecraft trajectory but are not considered in this work. For example, the dust size distribution may be variable and is difficult to constrain. The lower limit of dust size cannot be detected directly and the dust size may not even follow a simple power-law distribution. E ring dynamics simulations suggest that smaller dust particles acquire inclination faster than the larger ones (Juhász and Horányi, 2002), which implies that the size distribution most likely varies with the latitude as well. Figures 3 and 4 only demonstrate the average tendency of the spacecraft potential near $4 \mathrm{R}_{\mathrm{S}}$. More details need to be taken into account for reproducing the spacecraft potential measured during individual RPX/plume transversal.

One of the effects caused by the dust impacts onto the spacecraft is the reduction of $\Phi_{\text {sc }}$. Because dust impacts reduce the detection energy threshold, this effect cannot be ignored when interpreting thermal electron measurements when the spacecraft is charged negatively. However, the drawback is that the impact plasma may also be detected and "contaminate" the measurements, especially when the instrument is located at/facing toward the ram side of the spacecraft. This leads to another interesting aspect-the detectability of the dust-spacecraft impact plasma.

Given that the impact plasma is relatively cold (in the order of $1 \mathrm{eV}$ ), the instruments most likely to detect the dust-spacecraft impact plasma are the Cassini CAPS and LP/RPWS. Near Enceladus, the spacecraft is charged negatively. Part of the impact plasma ions are drawn back to the spacecraft with a few eV energy and may be detectable by the CAPS ion spectrometer. Similarly, the impact plasma may also be collected and contribute to the LP currentvoltage curve.

To be detected by the Cassini instruments, the impact plasma flux must be comparable to or higher than the ambient plasma. This condition is most likely the case in the plume of Enceladus, where the dust density is the highest. During Enceladus flybys in 2008, the CAPS ion spectra detected a low energy component $(<10 \mathrm{eV})$ whose timespans roughly coincide with those periods when the spacecraft flew through the plume (Tokar et al., 2009). These 
detections were interpreted as a southern-directed beam of ions accelerated by a electric field located at more than $400 \mathrm{~km}$ south of Enceladus (Farrell et al., 2010). An alternative explanation is that the low energy ion features are in fact associated with the impact plasma ions generated at the spacecraft surface. This could be verified by examining the correlation between the (low energy) ion intensity and the dust flux, or between their energy distribution and the spacecraft potential. The identification of the impact plasma in the in situ plasma measurements will provide important constraints in the impact plasma production rate, which is essential for estimating the influence of this "artificial" plasma in contaminating the thermal plasma data. Nonetheless, such analysis is outside the scope of this paper. The identification of dust-spacecraft impact plasma and the corresponding effects will be addressed in future works.

\section{Summary}

In this work we investigate the longitudinal and the latitudinal spacecraft potential variations near Enceladus. Due to the collection of ions from the dust-spacecraft impact plasma, our model suggests that the spacecraft is charged less negatively when traversing through dust-rich regions. The simulation results need to be systematically verified using the Cassini dust and plasma measurements. We also suggest to search for signatures of dust-spacecraft impact plasma in the Cassini plasma spectrometer (CAPS) and Langmuir probe (LP/RPWS) measurements.

Acknowledgments. The authors acknowledge the support from the Cassini project. The authors thank the anonymous reviewer for useful comments. The authors thank Paige Northway for assistance in improving the readability of the manuscript.

\section{References}

Dietzel, H., G. Eichhorn, H. Fechtig, E. Grun, H.-J. Hoffmann, and J. Kissel, The HEOS 2 and HELIOS micrometeoroid experiments, J. Phys. E Sci. Instrum., 6, 209-217, doi:10.1088/0022-3735/6/3/008, 1973.

Farrell, W. M., W. S. Kurth, D. A. Gurnett, R. E. Johnson, M. L. Kaiser, J. Wahlund, and J. H. Waite, Electron density dropout near Enceladus in the context of water-vapor and water-ice, Geophys. Res. Lett., 36, L10203, doi:10.1029/2008GL037108, 2009.

Farrell, W. M., W. S. Kurth, R. L. Tokar, J.-E. Wahlund, D. A. Gurnett, Z. Wang, R. J. MacDowall, M. W. Morooka, R. E. Johnson, and J. H. Waite, Modification of the plasma in the near-vicinity of Enceladus by the enveloping dust, Geophys. Res. Lett., 37, L20202, doi:10.1029/2010GL044768, 2010.

Goertz, C. K. and W. Ip, Limitation of electrostatic charging of dust particles in a plasma, Geophys. Res. Lett., 11, 349-352, doi:10.1029/GL011i004p00349, 1984.

Göller, J. R. and E. Grün, Calibration of the Galileo/Ulysses dust detectors with different projectile materials and at varying impact angles, Planet. Space Sci., 37, 1197-1206, 1989.

Grard, R. J. L. and J. K. E. Tunaley, Photoelectron sheath near a planar probe in interplanetary space, J. Geophys. Res., 76, 2498-2505, doi:10.1029/JA076i010p02498, 1971.

Gurnett, D. A., W. S. Kurth, D. L. Kirchner, G. B. Hospodarsky, T. F. Averkamp, P. Zarka, A. Lecacheux, R. Manning, A. Roux, P. Canu, N. Cornilleau-Wehrlin, P. Galopeau, A. Meyer, R. Boström, G. Gustafsson, J.-E. Wahlund, L. Åhlen, H. O. Rucker, H. P. Ladreiter, W. Macher, L. J. C. Woolliscroft, H. Alleyne, M. L. Kaiser, M. D. Desch, W. M. Farrell, C. C. Harvey, P. Louarn, P. J. Kellogg, K. Goetz, and A. Pedersen, The Cassini radio and plasma wave investigation, Space Sci. Rev., 114, 395-463, doi:10.1007/s11214-004-1434-0, 2004.

Gurnett, D. A., A. M. Persoon, W. S. Kurth, J. B. Groene, T. F. Averkamp, M. K. Dougherty, and D. J. Southwood, The variable rotation pe- riod of the inner region of Saturn's plasma disk, Science, 316, 442, doi:10.1126/science.1138562, 2007.

Gustafsson, G. and J.-E. Wahlund, Electron temperatures in Saturn's plasma disc, Planet. Space Sci., 58, 1018-1025, doi:10.1016/j.pss.2010.03.007, 2010.

Hansen, C. J., L. Esposito, A. I. F. Stewart, J. Colwell, A. Hendrix, W. Pryor, D. Shemansky, and R. West, Enceladus' water vapor plume, Science, 311, 1422-1425, doi:10.1126/science.1121254, 2006.

Havnes, O., T. K. Aanesen, and F. Melandso, On dust charges and plasma potentials in a dusty plasma with dust size distribution, J. Geophys. Res., 95, 6581-6585, doi:10.1029/JA095iA05p06581, 1990.

Horányi, M., Charged dust dynamics in the solar system, Ann. Rev. Astrophys., 34, 383-418, doi:10.1146/annurev.astro.34.1.383, 1996.

Hornung, K. and M. Drapatz, Residual ionization after impact of large dust particles, in The Comet Hallay Probe Plasma Environment, p. 23-37, European Space Agency, 1979.

Hsu, H.-W., M. Horányi, S. Kempf, and E. Grün, Spacecraft charging near Enceladus, Geophys. Res. Lett., 39, L06108, doi:10.1029/2012GL050999, 2012.

Juhász, A. and M. Horányi, Saturn's E ring: A dynamical approach, $J$. Geophys. Res., 107, 1-10, doi:10.1029/2001JA000182, 2002.

Kempf, S., U. Beckmann, R. Srama, M. Horanyi, S. Auer, and E. Grün, The electrostatic potential of E ring particles, Planet. Space Sci., 54, 999-1006, doi:10.1016/j.pss.2006.05.012, 2006.

Kempf, S., U. Beckmann, G. Moragas-Klostermeyer, F. Postberg, R. Srama, T. Economou, J. Schmidt, F. Spahn, and E. Grün, The E ring in the vicinity of Enceladus I: Spatial distribution and properties of the ring particles, Icarus, 193, 420-437, doi:10.1016/j.icarus.2007.06.027, 2008.

Kempf, S., R. Srama, E. Grün, A. Mocker, F. Postberg, J. K. Hillier, M. Horányi, Z. Sternovsky, B. Abel, A. Beinsen, R. Thissen, J. Schmidt, F. Spahn, and N. Altobelli, Linear high resolution dust mass spectrometer for a mission to the Galilean satellites, Planet. Space Sci., 65, 10-20, doi:10.1016/j.pss.2011.12.019, 2012.

Lewis, G. R., N. André, C. S. Arridge, A. J. Coates, L. K. Gilbert, D. R. Linder, and A. M. Rymer, Derivation of density and temperature from the Cassini Huygens CAPS electron spectrometer, Planet. Space Sci., 56, 901-912, doi:10.1016/j.pss.2007.12.017, 2008.

Morooka, M. W., J.-E. Wahlund, A. I. Eriksson, W. M. Farrell, D. A. Gurnett, W. S. Kurth, A. M. Persoon, M. Shafiq, M. André, and M. K. G. Holmberg, Dusty plasma in the vicinity of Enceladus, J. Geophys. Res., 116, A12221, doi:10.1029/2011JA017038, 2011.

Mott-Smith, H. M. and I. Langmuir, The theory of collectors in gaseous discharges, Phys. Rev., 28, 727-763, doi:10.1103/PhysRev.28.727, 1926.

Olson, J., W. J. Miloch, S. Ratynskaia, and V. Yaroshenko, Potential structure around the Cassini spacecraft near the orbit of Enceladus, Phys. Plasmas, 17(10), 102,904, doi:10.1063/1.3486523, 2010.

Persoon, A. M., D. A. Gurnett, W. S. Kurth, and J. B. Groene, A simple scale height model of the electron density in Saturn's plasma disk, Geophys. Res. Lett., 33, L18106, doi:10.1029/2006GL027090, 2006.

Porco, C. C., P. Helfenstein, P. C. Thomas, A. P. Ingersoll, J. Wisdom, R. West, G. Neukum, T. Denk, R. Wagner, T. Roatsch, S. Kieffer, E. Turtle, A. McEwen, T. V. Johnson, J. Rathbun, J. Veverka, D. Wilson, J. Perry, J. Spitale, A. Brahic, J. A. Burns, A. D. DelGenio, L. Dones, C. D. Murray, and S. Squyres, Cassini observes the active south pole of Enceladus, Science, 311, 1393-1401, doi:10.1126/science.1123013, 2006.

Shafiq, M., J.-E. Wahlund, M. W. Morooka, W. S. Kurth, and W. M. Farrell, Characteristics of the dust-plasma interaction near Enceladus' South Pole, Planet. Space Sci., 59, 17-25, doi:10.1016/j.pss.2010.10.006, 2011.

Sittler, E. C., M. Thomsen, D. Chornay, M. D. Shappirio, D. Simpson, R. E. Johnson, H. T. Smith, A. J. Coates, A. M. Rymer, F. Crary, D. J. McComas, D. T. Young, D. Reisenfeld, M. Dougherty, and N. Andre, Preliminary results on Saturn's inner plasmasphere as observed by Cassini: Comparison with Voyager, Geophys. Res. Lett., 32, L14S07, 2005.

Sittler, E. C., M. Thomsen, R. E. Johnson, R. E. Hartle, M. Burger, D. Chornay, M. D. Shappirio, D. Simpson, H. T. Smith, A. J. Coates, A. M. Rymer, D. J. McComas, D. T. Young, D. Reisenfeld, M. Dougherty, and N. Andre, Cassini observations of Saturn's inner plasmasphere: Saturn orbit insertion results, Planet. Space Sci., 54, 1197-1210, doi:10.1016/j.pss.2006.05.038, 2006.

Spahn, F., J. Schmidt, N. Albers, M. Hörning, M. Makuch, M. Seiß, S. Kempf, R. Srama, V. Dikarev, S. Helfert, G. Moragas-Klostermeyer, 
A. V. Krivov, M. Sremčević, A. J. Tuzzolino, T. Economou, and E. Grün, Cassini dust measurements at Enceladus and implications for the origin of the E ring, Science, 311, 1416-1418, doi:10.1126/science.1121375, 2006.

Srama, R., T. J. Ahrens, N. Altobelli, S. Auer, J. G. Bradley, M. Burton, V. V. Dikarev, T. Economou, H. Fechtig, M. Görlich, M. Grande, A. Graps, E. Grün, O. Havnes, S. Helfert, M. Horanyi, E. Igenbergs, E. K. Jessberger, T. V. Johnson, S. Kempf, A. V. Krivov, H. Krüger, A. Mocker-Ahlreep, G. Moragas-Klostermeyer, P. Lamy, M. Landgraf, D. Linkert, G. Linkert, F. Lura, J. A. M. McDonnell, D. Möhlmann, G. E. Morfill, M. Müller, M. Roy, G. Schäfer, G. Schlotzhauer, G. H. Schwehm, F. Spahn, M. Stübig, J. Svestka, V. Tschernjawski, A. J. Tuzzolino, R. Wäsch, and H. A. Zook, The Cassini cosmic dust analyzer, Space Sci. Rev., 114, 465-518, doi:10.1007/s11214-004-1435-z, 2004.

Srama, R., S. Kempf, G. Moragas-Klostermeyer, S. Helfert, T. J. Ahrens, N. Altobelli, S. Auer, U. Beckmann, J. G. Bradley, M. Burton, V. V. Dikarev, T. Economou, H. Fechtig, S. F. Green, M. Grande, O. Havnes, J. K. Hillier, M. Horanyi, E. Igenbergs, E. K. Jessberger, T. V. Johnson, H. Krüger, G. Matt, N. McBride, A. Mocker, P. Lamy, D. Linkert, G. Linkert, F. Lura, J. A. M. McDonnell, D. Möhlmann, G. E. Morfill, F. Postberg, M. Roy, G. H. Schwehm, F. Spahn, J. Svestka, V. Tschernjawski, A. J. Tuzzolino, R. Wäsch, and E. Grün, In situ dust measurements in the inner Saturnian system, Planet. Space Sci., 54, 967-987, doi:10.1016/j.pss.2006.05.021, 2006.

Tokar, R. L., R. E. Johnson, M. F. Thomsen, R. J. Wilson, D. T. Young, F. J. Crary, A. J. Coates, G. H. Jones, and C. S. Paty, Cassini detection of Enceladus' cold water-group plume ionosphere, Geophys. Res. Lett., 36, L13203, doi:10.1029/2009GL038923, 2009.

Wahlund, J.-E., R. Boström, G. Gustafsson, D. A. Gurnett, W. S. Kurth, T. Averkamp, G. B. Hospodarsky, A. M. Persoon, P. Canu, A. Pedersen, M. D. Desch, A. I. Eriksson, R. Gill, M. W. Morooka, and M. André, The inner magnetosphere of Saturn: Cassini RPWS cold plasma results from the first encounter, Geophys. Res. Lett., 32, L20S09, doi:10.1029/2005GL022699, 2005.

Wahlund, J.-E., M. André, A. I. E. Eriksson, M. Lundberg, M. W. Morooka, M. Shafiq, T. F. Averkamp, D. A. Gurnett, G. B. Hospodarsky, W. S. Kurth, K. S. Jacobsen, A. Pedersen, W. Farrell, S. Ratynskaia, and N. Piskunov, Detection of dusty plasma near the E-ring of Saturn, Planet. Space Sci., 57, 1795-1806, doi:10.1016/j.pss.2009.03.011, 2009.

Waite, J. H., M. R. Combi, W.-H. Ip, T. E. Cravens, R. L. McNutt, W. Kasprzak, R. Yelle, J. Luhmann, H. Niemann, D. Gell, B. Magee, G. Fletcher, J. Lunine, and W.-L. Tseng, Cassini ion and neutral mass spectrometer: Enceladus plume composition and structure, Science, 311, 1419-1422, doi:10.1126/science.1121290, 2006.

Warwick, J. W., J. B. Pearce, D. R. Evans, T. D. Carr, J. J. Schauble, J. K. Alexander, M. L. Kaiser, M. D. Desch, M. Pedersen, A. Lecacheux, G. Daigne, A. Boischot, and C. H. Barrow, Planetary radio astronomy observations from Voyager 1 near Saturn, Science, 212, 239-243, doi:10.1126/science.212.4491.239, 1981.

Whipple, E. C., Potentials of surfaces in space, Rep. Prog. Phys., 44, 1197 $1250,1981$.

Yaroshenko, V. V., S. Ratynskaia, J. Olson, N. Brenning, J. Wahlund, M. Morooka, W. S. Kurth, D. A. Gurnett, and G. E. Morfill, Characteristics of charged dust inferred from the Cassini RPWS measurements in the vicinity of Enceladus, Planet. Space Sci., 57, 1807-1812, doi:10.1016/j.pss.2009.03.002, 2009.

Young, D. T., J. J. Berthelier, M. Blanc, J. L. Burch, A. J. Coates, R. Goldstein, M. Grande, T. W. Hill, R. E. Johnson, V. Kelha, D. J. McComas, E. C. Sittler, K. R. Svenes, K. Szegö, P. Tanskanen, K. Ahola, D. Anderson, S. Bakshi, R. A. Baragiola, B. L. Barraclough, R. K. Black, S. Bolton, T. Booker, R. Bowman, P. Casey, F. J. Crary, D. Delapp, G. Dirks, N. Eaker, H. Funsten, J. D. Furman, J. T. Gosling, H. Hannula, C. Holmlund, H. Huomo, J. M. Illiano, P. Jensen, M. A. Johnson, D. R. Linder, T. Luntama, S. Maurice, K. P. McCabe, K. Mursula, B. T. Narheim, J. E. Nordholt, A. Preece, J. Rudzki, A. Ruitberg, K. Smith, S. Szalai, M. F. Thomsen, K. Viherkanto, J. Vilppola, T. Vollmer, T. E. Wahl, M. Wüest, T. Ylikorpi, and C. Zinsmeyer, Cassini plasma spectrometer investigation, Space Sci. Rev., 114, 1-112, doi:10.1007/s11214-004-1406-4, 2004.

H.-W. Hsu (e-mail: sean.hsu@lasp.colorado.edu), M. Horányi, and S. Kempf 